\title{
Language supplementary motor area syndrome correlated with dynamic changes in perioperative task-based functional MRI activations: case report
}

\author{
Jaime A. Quirarte, BA, ${ }^{1}$ Vinodh A. Kumar, MD, ${ }^{2}$ Ho-Ling Liu, PhD, ${ }^{3}$ Kyle R. Noll, PhD, ${ }^{4}$ \\ Jeffrey S. Wefel, PhD, ${ }^{4}$ and Frederick F. Lang, MD ${ }^{1}$
}

Departments of ${ }^{1}$ Neurosurgery, ${ }^{2}$ Neuroradiology, and ${ }^{3}$ maging Physics, University of Texas MD Anderson Cancer Center; and ${ }^{4}$ Department of Neuro-Oncology, Section of Neuropsychology, University of Texas MD Anderson Cancer Center, Houston, Texas

\begin{abstract}
Supplementary motor area (SMA) syndrome is well known; however, the mechanism underlying recovery from language SMA syndrome is unclear. Herein the authors report the case of a right-handed woman with speech aphasia following resection of an oligodendroglioma located in the anterior aspect of the left superior frontal gyrus. The patient exhibited language SMA syndrome, and functional MRI (fMRI) findings 12 days postoperatively demonstrated a complete shift of blood oxygen level-dependent (BOLD) activation to the contralateral right language SMA/pre-SMA as well as coequal activation and an increased volume of activation in the left Broca's area and the right Broca's homolog. The authors provide, to the best of their knowledge, the first description of dynamic changes in task-based hemispheric language BOLD $\mathrm{fMRI}$ activations across the preoperative, immediate postoperative, and more distant postoperative settings associated with the development and subsequent complete resolution of the clinical language SMA syndrome.
\end{abstract}

https://thejns.org/doi/abs/10.3171/2020.4.JNS193250

KEYWORDS functional MRI; Broca's area; Broca's homolog; language supplementary motor area syndrome; reorganization; oncology

0 UPPLEMENTARY motor area (SMA) syndrome, a wellknown operative risk associated with resecting lesions near or within the posterior aspect of the superior frontal gyrus, is characterized by contralateral hemiparesis and/or hemiplegia with various degrees of speech deficits in the language-dominant hemisphere, ranging from speech hesitancy to complete mutism. ${ }^{1}$ Recovery typically occurs within a few weeks to months after surgery. ${ }^{1-4}$ While the syndrome has been extensively described, the mechanism underlying the recovery of language in SMA syndrome is unclear.

It has been shown that task-based functional MRI (fMRI) can localize the pre-SMA when language paradigms are performed. ${ }^{5,6}$ Nelson et al. found that there is a $100 \%$ risk for a language SMA syndrome when a resection is within $5 \mathrm{~mm}$ of the pre-SMA blood oxygen level-dependent (BOLD) fMRI activation.7 However, the evolution of fMRI activations during recovery of the language component of the SMA syndrome has yet to be described. We report the case of a young woman with SMA-related speech aphasia following resection of an oligodendroglioma in the left superior frontal gyrus.

\section{Case Report}

History and Examination

A 23-year-old, right-handed college-educated woman presented with alternating headaches, visual disturbances, and occasional difficulty speaking. Electroencephalography performed at an outside institution revealed normal findings. Preoperative neuropsychological evaluation at our institution indicated isolated impairment with aspects of working memory $(\mathrm{z}=-1.62)$. Language and motor functions were within normative expectations.

\section{MRI Acquisition}

fMRI, diffusion tensor imaging (DTI), and structural MRI were performed 4 times, including preoperative imaging and 3 follow-up studies at 12, 89, and 138 days postoperation, using a 3-T MRI scanner (GE Healthcare) with

ABBREVIATIONS BOLD = blood oxygen level-dependent; DTI = diffusion tensor imaging; fMRI = functional MRI; SMA = supplementary motor area .

SUBMITTED January 24, 2020. ACCEPTED April 3, 2020.

INCLUDE WHEN CITING Published online June 5, 2020; DOI: 10.3171/2020.4.JNS193250. 
an 8-channel head coil. Functional images were acquired using a T2*-weighted gradient echo, echo planar imaging sequence (TR $2000 \mathrm{msec}$, TE $25 \mathrm{msec}$, matrix size $64 \times$ 64 , FOV $24 \times 24 \mathrm{~cm}$, slice thickness $4 \mathrm{~mm}$ with no gap, 32 slices to cover the entire brain).

\section{fMRI Paradigms}

Two fMRI covert speech paradigms were used: letter fluency and category fluency. Both paradigms were block design with alternating active and control blocks. Each paradigm was 4 minutes 20 seconds, with blocks alternating for 20 seconds each. In the active block, the participant was presented with a letter of the alphabet or category (e.g., cities, types of food, colors) and asked to covertly generate words that started with the letter or category. In the control phase, the participant was asked to tap her index finger on her thumb bilaterally for both paradigms.

During functional imaging acquisition, each paradigm was displayed using an MRI-compatible, 32-inch-wide liquid crystal display (Invivo SensaVue, Philips).

\section{Image Analysis}

Image analysis was performed using the DynaSuite Neuro software version 3.0 (Invivo, Philips). Image preprocessing for fMRI included motion correction and spatial smoothing with a 4-mm full width at half maximum gaussian kernel. A functional activation map was created using the correlation analysis of the task paradigms convolved with a canonical hemodynamic response function and the signal intensity time course for each voxel. A statistical threshold of $t=6.3\left(p<10^{-4}\right.$, corrected) was applied to optimize the visualization of language areas based on the preoperative study and to standardize thresholding across the 4 fMRI studies.

\section{Laterality Calculation}

Laterality index was calculated using the following formula: (activation volume left - activation volume right $_{\text {f }} /$ / (activation volume $e_{\text {left }}+$ activation volume $_{\text {right }}$ ). The fMRI result is defined as codominant if the laterality index falls between -0.2 to +0.2 , right dominant if less than -0.2 , and left dominant if greater than $+0.2{ }^{8}$

\section{Neuropsychological Evaluation}

Testing was conducted by a neuropsychologist as part of comprehensive pre- and postoperative neuropsychological evaluations for clinical purposes. Testing occurred at 3 times closely yoked to the first 3 fMRI studies. Measures utilized included the Repeatable Battery for the Assessment of Neuropsychological Status (12 days postoperatively only) as well as those utilized in our prior studies. ${ }^{9}$ Test scores were standardized using published normative data, stratified by patient age, handedness, sex, and level of education and converted into z scores (mean 0, SD 1). Per common convention, z scores were considered impaired if they fell at or below -1.5 .

\section{Initial Imaging and Surgical Treatment}

Preoperative brain MRI demonstrated a nonenhancing infiltrating mass within the left superior frontal gyrus
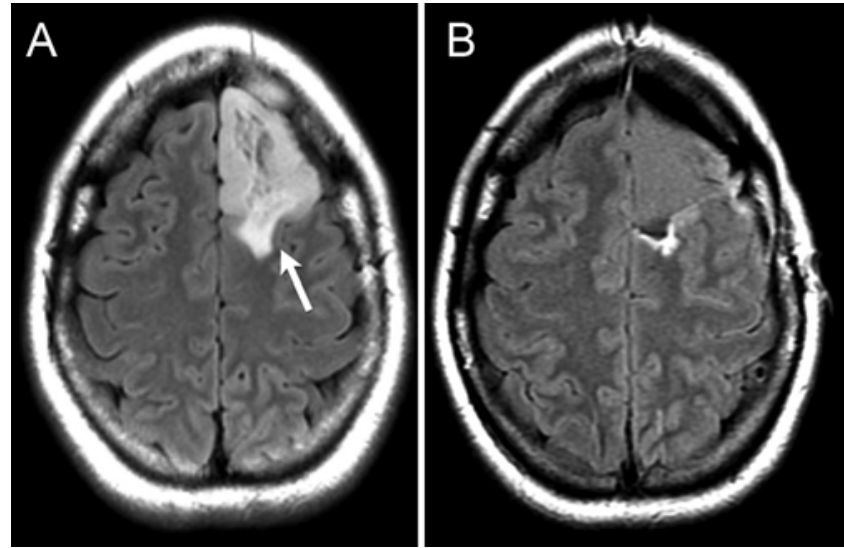

FIG. 1. Preoperative axial FLAIR MR image demonstrated an infiltrative glioma (arrow) involving the left superior frontal gyrus (A). Postoperative axial FLAIR MR image revealed gross-total resection of the pathologically proven oligodendroglioma (B).

suggestive of a low-grade glioma (Fig. 1A). Preoperative fMRI expressive language mapping was performed, showing left hemisphere language dominance in this strongly right-handed patient (Edinburgh Handedness Inventory +80 ). The fMRI also demonstrated activation of the left pre-SMA (Fig. 2A), which was posteriorly displaced and abutted the glioma. To a lesser extent, activation was also noted in the right pre-SMA.

The patient elected to undergo an awake left frontal craniotomy for resection of the mass. Intraoperatively, inspection of the brain revealed an expanded left superior frontal gyrus, and computer-assisted stereotactic technology was used to outline the edges of the mass. The superior frontal sulcus defined the lateral border of the glioma and another sulcus defined the posterior border. Intraoperative cortical mapping using phase reversal ensured the tumor was a safe distance from the motor cortex. Language mapping was not performed. En bloc resection of the glioma was achieved.

\section{Postoperative Course and Follow-Up Imaging}

Immediately after surgery, the patient had normal strength in all extremities and was able to understand language and carry out complex commands. However, she exhibited a significant speech aphasia and was unable to initiate spontaneous speech, consistent with a language SMA syndrome.

Immediate postoperative brain MRI (within 24 hours) demonstrated gross-total resection of the glioma (Fig. 1B). Pathology revealed a low-grade oligodendroglioma (IDH mutant, $1 \mathrm{p} / 19 \mathrm{q}$ co-deleted). There were no pericavity ischemic changes on diffusion-weighted imaging. Electroencephalography performed 4 days postoperatively revealed no seizure activity. Intravenous Keppra $500 \mathrm{mg}$ twice a day was administered prophylactically for 4 days postoperatively. Dexamethasone was administered postoperatively as follows: $6 \mathrm{mg}$ intravenously every 6 hours for 4 days and $6 \mathrm{mg}$ by mouth every 6 hours on the 5th and 6 th postoperative days, then tapered.

Inpatient neuropsychological evaluation was performed 5 days after surgery. This showed severe aphasia, predom- 

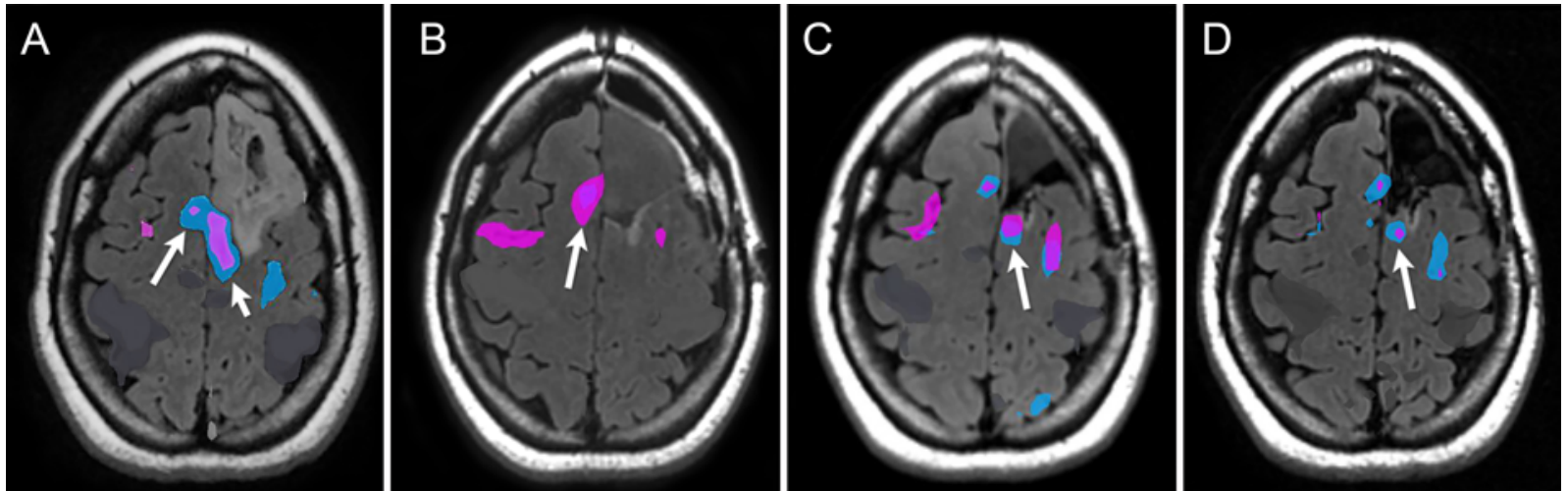

FIG. 2. Axial fMRI of bilateral pre-SMAs at 4 timepoints revealed shifts in hemispheric BOLD activation. Image obtained 7 days prior to surgery (A) demonstrated increased activation of the left pre-SMA (small arrow) for both category fluency (pink) and letter fluency (blue) paradigms. To a lesser extent, there was also activation in the right pre-SMA (long arrow). Image obtained 12 days postoperatively (B) showing fMRI activation completely shifted to the right pre-SMA (arrow). Images obtained 89 days (C) and 138 days (D) postoperatively showing the return of fMRI activation in the left pre-SMA (arrows).

inantly expressive in nature, with severely impaired $(\mathrm{z}<$ $-3.00)$ confrontation naming including semantic paraphasic errors, and severely impaired phonemic and semantic verbal fluency. However, she was able to form simple sentences, and her comprehension was relatively preserved as demonstrated by her ability to understand task instructions. Impairments were also observed in verbal learning and memory, auditory attention, and processing speed. Overall, the findings were consistent with the disruption of left-hemisphere language function with particular indication of dysfunction within the expressive language network.

To examine potential alterations in fMRI activations during the language SMA syndrome, the patient underwent an additional fMRI study 12 days after surgery. As depicted in Fig. 2B, there was no BOLD activation in the left pre-SMA and an increased volume of BOLD activation in the contralateral right pre-SMA. In addition, the patient had coactivation and an increased volume of activation in the left Broca's area and, interestingly, in the right Broca's homolog (Fig. 3B). The left frontal aslant tract was intact postoperatively as confirmed with DTI.

Follow-up neuropsychological evaluation was again conducted 88 days after surgery to assess the patient's recovery trajectory. At this juncture, neurocognitive functioning was broadly within normal limits across all domains including language abilities, indicating full recovery from the language SMA syndrome and a return to the preoperative baseline level of cognitive functioning.

To evaluate potential functional reorganization underlying her neurocognitive recovery, follow-up fMRI was performed at 89 days postoperatively (Figs. $2 \mathrm{C}$ and $3 \mathrm{C}$ ) and again at 138 days postoperatively (Figs. 2D and 3D). At both times, fMRI showed the return of BOLD activation to the left pre-SMA (Fig. 2C and D) with a coinciding decrease in activation volume in the right pre-SMA. In
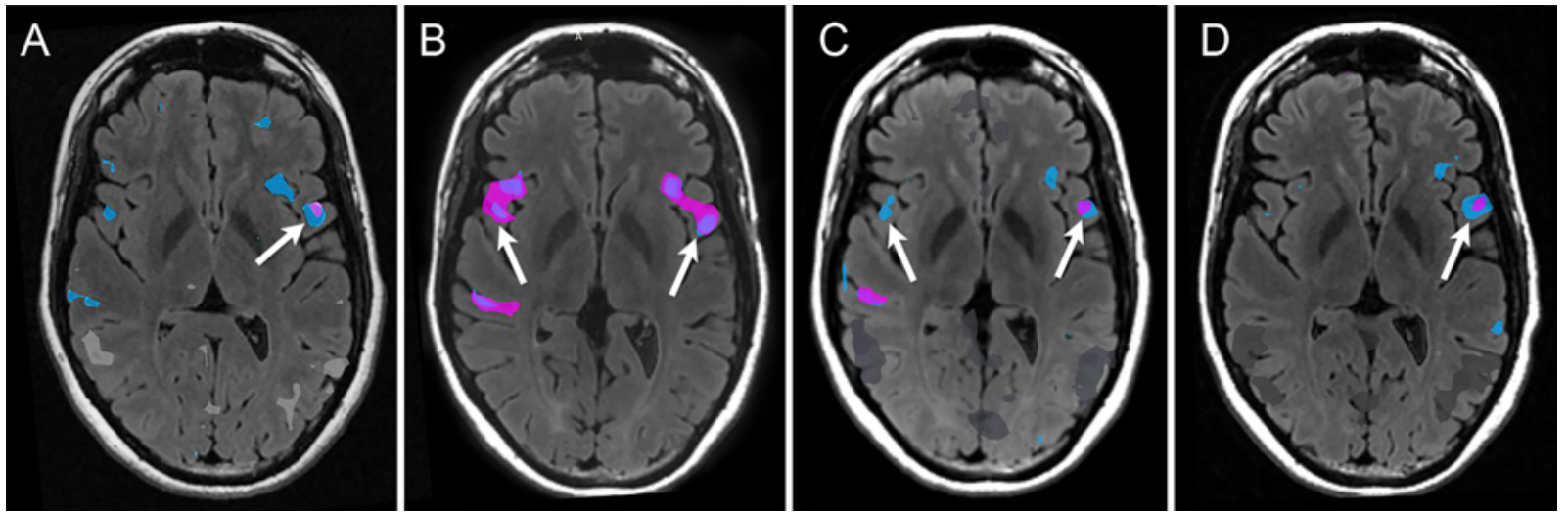

FIG. 3. Axial fMRI of Broca's area at 4 timepoints revealed shifts in hemispheric BOLD activation. A: Image obtained 7 days prior to surgery demonstrating left-hemisphere-dominant Broca's area fMRI activation (arrow). B: Image obtained 12 days postoperatively showing coactivation and an increased volume of BOLD activation in both the left Broca's area and the right Broca's homolog during the clinical language SMA syndrome (arrows). C: Image obtained 89 days postoperatively showing bilateral fMRI activations in the left Broca's area and right Broca's homolog, though to a lesser extent than the 12-day postoperative study. D: Image obtained 138 days postoperatively essentially showing only left Broca's area activation. Clinically, the patient had completely recovered from the language SMA syndrome by the 89th postoperative day. 


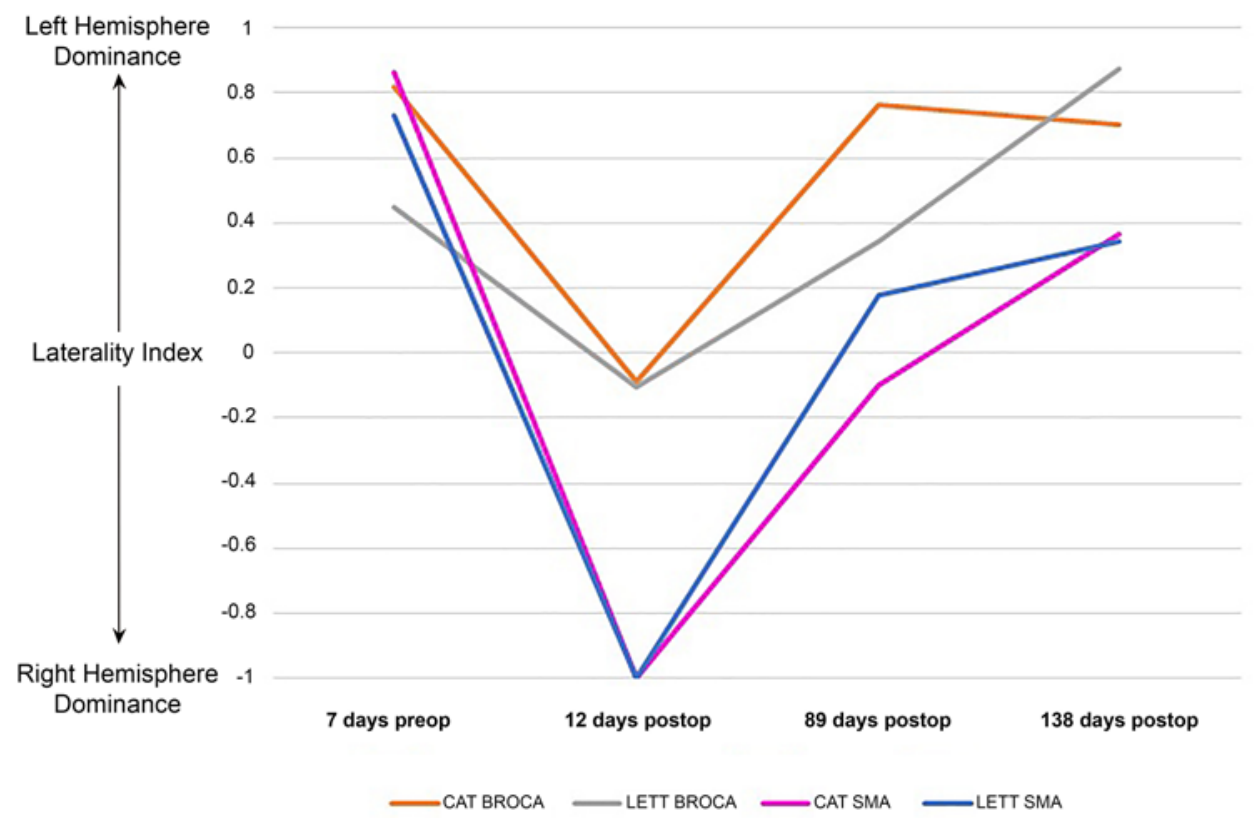

FIG. 4. Laterality index calculation at 4 timepoints for category fluency (CAT) and letter fluency (LETT) paradigms for the bilateral pre-SMAs, left Broca's area, and right Broca's homolog. Note that values on the $y$-axis range from -1 to 1 , with values of -1 to -0.2 indicating right-hemisphere dominance, -0.2 to +0.2 indicating codominance, and +0.2 to +1 indicating left-hemisphere dominance.

addition, the last fMRI study showed a return of dominant BOLD activation in the left Broca's area (Figs. 3D and 4).

\section{Discussion}

Language SMA syndrome is classically described as aphasia with various levels of severity following resection near or in the SMA. ${ }^{10,11}$ The medial frontal cortex is somatographically organized into pre-SMA and SMA regions..$^{12}$ In healthy subjects, fMRI shows BOLD activation in the dominant-hemisphere pre-SMA with linguistic operations. ${ }^{13,14}$ The pre-SMA activations are more anteriorly situated with language tasks than with finger-tapping tasks. ${ }^{6}$ In our case, preoperative fMRI showed an infiltrative glioma directly abutting the anterior aspect of the left pre-SMA. The patient developed a language SMA syndrome postoperatively, and the case demonstrates unique hemispheric fMRI activation patterns during recovery from the language SMA syndrome.

Analogous to our case, it has been shown that lesions contacting the SMA are associated with increased BOLD fMRI activation in the contralateral SMA. ${ }^{15}$ It has also been shown that increased preoperative activation in the pre-SMA contralateral to the lesion is associated with an increased risk of postoperative speech deficit. ${ }^{3} \mathrm{fMRI}$ at 12 days postoperatively showed a complete shift of BOLD activation to the contralateral right pre-SMA (Fig. 2B) as well as an increase in volume and equal activation of the left Broca's area and the right Broca's homolog (Fig. 3B). Given the preoperative fMRI findings, the right Broca's homolog likely has no "functional" role in language in this patient. At the third fMRI study 89 days after surgery, both pre-SMAs showed coactivations (Fig. 4), and at the fourth fMRI study 138 days postoperatively, the pre-SMA activations trended toward left hemisphere dominance. There was a return to left-hemisphere-dominant Broca's area BOLD activation at 89 and 138 days postoperatively.

Chivukula et al. reported a language SMA syndrome and recovery with reorganization as evidenced by BOLD activation in the contralateral pre-SMA in 2 patients. ${ }^{16}$ In one of the patients, they attributed the right pre-SMA reorganization to partial resection of the left pre-SMA during removal of an oligodendroglioma (fMRI performed 64 months postoperatively). In the other patient, they ascribed right pre-SMA reorganization to resection of the left frontal aslant tract (fMRI performed 32 months postoperatively). The frontal aslant tract is a white matter tract that connects the ipsilateral pre-SMA to the ipsilateral Broca's area. ${ }^{16,17}$ It has been found to be highly associated with deficits in articulatory planning and speech initiation with direct electrical stimulation..$^{18}$ In our case, the left pre-SMA was not resected and DTI demonstrated that the left frontal aslant tract was intact. This may account for the "temporary," as opposed to "permanent," reorganization of fMRI activation to the right pre-SMA. In another study, Baker et al. described a previously unknown part of the frontal aslant tract that passes through the corpus callosum to connect the ipsilateral premotor area to the contralateral SMA and premotor area. ${ }^{19}$ They proposed that this tract maintains interhemispheric connectivity between these regions and thus may serve as a potential mechanism of recovery from language SMA syndrome.

We noted increased volume and equal activation of the BOLD signal in the left Broca's area and the right Broca's homolog during the SMA syndrome. We hypothesize that the right Broca's homolog activation is attributable to an 
attempted "futile" compensation, which may temporarily interfere with language SMA recovery. This activation pattern is particularly evident on the category fluency paradigm at 12 days postoperatively (Fig. 3B) coinciding with the speech aphasia.

Our findings are somewhat analogous to those of Sato et al., who found that individuals who stutter lack functional lateralization for auditory speech processing, as opposed to nonstutterers, who display left-hemisphere dominance for phonemic stimuli. ${ }^{20}$

It can be argued that the lack of left pre-SMA activity observed 12 days postoperatively may be attributed to postsurgical changes at the resection cavity near the SMA, which resulted in neurovascular uncoupling of the BOLD response; however, this alone does not explain the other BOLD activation patterns in this case.

\section{Conclusions}

This case report demonstrates novel pre- and postoperative fMRI findings in the development and recovery of a pure language SMA syndrome. A larger sample size and prospective studies are needed to better elucidate the mechanism of recovery from a language SMA syndrome using task-based fMRI and to establish improved predictors of recovery. Further studies using resting-state functional connectivity analyses in the perioperative period may also be useful.

\section{References}

1. Rostomily RC, Berger MS, Ojemann GA, Lettich E. Postoperative deficits and functional recovery following removal of tumors involving the dominant hemisphere supplementary motor area. J Neurosurg. 1991;75(1):62-68.

2. Bannur U, Rajshekhar V. Post operative supplementary motor area syndrome: clinical features and outcome. $\mathrm{Br} \mathrm{J} \mathrm{Neu-}$ rosurg. 2000;14(3):204-210.

3. Krainik A, Lehéricy S, Duffau H, et al. Postoperative speech disorder after medial frontal surgery: role of the supplementary motor area. Neurology. 2003;60(4):587-594.

4. Peraud A, Meschede M, Eisner W, et al. Surgical resection of grade II astrocytomas in the superior frontal gyrus. Neurosurgery. 2002;50(5):966-977.

5. Crosson B, Sadek JR, Maron L, et al. Relative shift in activity from medial to lateral frontal cortex during internally versus externally guided word generation. J Cogn Neurosci. 2001;13(2):272-283.

6. Hiroshima S, Anei R, Murakami N, Kamada K. Functional localization of the supplementary motor area. Neurol Med Chir (Tokyo). 2014;54(7):511-520.

7. Nelson L, Lapsiwala S, Haughton VM, et al. Preoperative mapping of the supplementary motor area in patients harboring tumors in the medial frontal lobe. J Neurosurg. 2002;97(5):1108-1114.

8. Lyo JK, Arevalo-Perez J, Petrovich Brennan N, et al. Preoperative fMRI localization of the supplementary motor area and its relationship with postoperative speech deficits. Neuroradiol J. 2015;28(3):281-288.
9. Wefel JS, Noll KR, Rao G, Cahill DP. Neurocognitive function varies by IDH1 genetic mutation status in patients with malignant glioma prior to surgical resection. Neuro Oncol. 2016;18(12):1656-1663.

10. Masdeu JC, Schoene WC, Funkenstein H. Aphasia following infarction of the left supplementary motor area: a clinicopathologic study. Neurology. 1978;28(12):1220-1223.

11. Vassal M, Charroud C, Deverdun J, et al. Recovery of functional connectivity of the sensorimotor network after surgery for diffuse low-grade gliomas involving the supplementary motor area. J Neurosurg. 2017;126(4):1181-1190.

12. Kim JH, Lee JM, Jo HJ, et al. Defining functional SMA and pre-SMA subregions in human MFC using resting state fMRI: functional connectivity-based parcellation method. Neuroimage. 2010;49(3):2375-2386.

13. Alario FX, Chainay H, Lehericy S, Cohen L. The role of the supplementary motor area (SMA) in word production. Brain Res. 2006;1076(1):129-143.

14. Huang J, Carr TH, Cao Y. Comparing cortical activations for silent and overt speech using event-related fMRI. Hum Brain Mapp. 2002;15(1):39-53.

15. Sailor J, Meyerand ME, Moritz CH, et al. Supplementary motor area activation in patients with frontal lobe tumors and arteriovenous malformations. AJNR Am J Neuroradiol. 2003;24(9):1837-1842.

16. Chivukula S, Pikul BK, Black KL, et al. Contralateral functional reorganization of the speech supplementary motor area following neurosurgical tumor resection. Brain Lang. 2018:183:41-46.

17. Catani M, Mesulam MM, Jakobsen E, et al. A novel frontal pathway underlies verbal fluency in primary progressive aphasia. Brain. 2013;136(Pt 8):2619-2628.

18. Kinoshita M, de Champfleur NM, Deverdun J, et al. Role of fronto-striatal tract and frontal aslant tract in movement and speech: an axonal mapping study. Brain Struct Funct. 2015;220(6):3399-3412.

19. Baker CM, Burks JD, Briggs RG, et al. The crossed frontal aslant tract: a possible pathway involved in the recovery of supplementary motor area syndrome. Brain Behav. 2018;8(3):e00926.

20. Sato Y, Mori K, Koizumi T, et al. Functional lateralization of speech processing in adults and children who stutter. Front Psychol. 2011;2:70.

\section{Disclosures}

Dr. Wefel is a consultant for Angiochem, Bayer, Juno, Novocure, and Vanquish Oncology.

\section{Author Contributions}

Conception and design: Kumar, Quirarte, Liu, Lang. Acquisition of data: all authors. Analysis and interpretation of data: all authors. Drafting the article: Kumar, Quirarte. Critically revising the article: all authors. Reviewed submitted version of manuscript: all authors. Approved the final version of the manuscript on behalf of all authors: Kumar. Study supervision: Kumar, Lang.

\section{Correspondence}

Vinodh A. Kumar: University of Texas MD Anderson Cancer Center, Houston, TX. vakumar@mdanderson.org. 